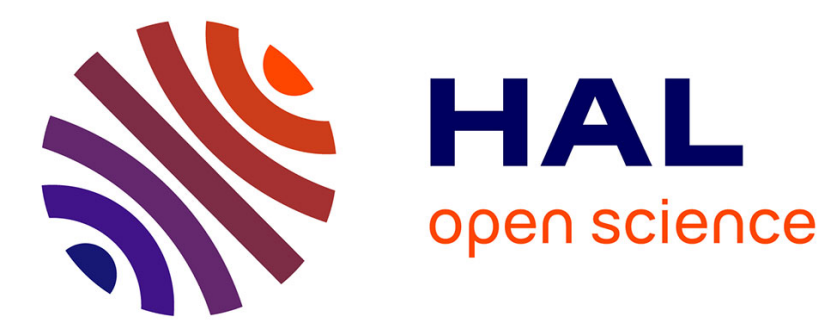

\title{
A general approach to securely querying XML
}

\author{
Ernesto Damiani, M. Fansi, Alban Gabillon, Stefania Marrara
}

\section{To cite this version:}

Ernesto Damiani, M. Fansi, Alban Gabillon, Stefania Marrara. A general approach to securely querying XML. Computer Standards and Interfaces, 2008, 30 (6), pp.379-389. 10.1016/j.csi.2008.03.006 . hal-00994104

\section{HAL Id: hal-00994104 \\ https://hal.science/hal-00994104}

Submitted on 26 May 2014

HAL is a multi-disciplinary open access archive for the deposit and dissemination of scientific research documents, whether they are published or not. The documents may come from teaching and research institutions in France or abroad, or from public or private research centers.
L'archive ouverte pluridisciplinaire HAL, est destinée au dépôt et à la diffusion de documents scientifiques de niveau recherche, publiés ou non, émanant des établissements d'enseignement et de recherche français ou étrangers, des laboratoires publics ou privés. 


\title{
A general approach to securely querying XML
}

\author{
Ernesto Damiani $^{\text {a }}$, Majirus Fansi ${ }^{\mathrm{b}}$, Alban Gabillon ${ }^{\mathrm{b}}$, Stefania Marrara ${ }^{\mathrm{a}, *}$ \\ ${ }^{a}$ Università degli Studi di Milano, Dipartimento di Tecnologie dell'Informazione via Bramante 6526013 Crema (CR), Italy \\ ${ }^{\mathrm{b}}$ Université de Pau et des Pays de l'Adour, IUT des Pays de l'Adour 40000 Mont-de-Marsan, France
}

Available online 8 March 2008

\begin{abstract}
XML access control requires the enforcement of highly expressive access control policies to support schema-, document and object-specific protection requirements. Access control models for XML data can be classified in two major categories: node filtering and query rewriting systems. The first category includes approaches that use access policies to compute secure user views on XML data sets. User queries are then evaluated on those views. In the second category of approaches, authorization rules are used to transform user queries to be evaluated against the original XML data set. The pros and cons for these approaches have been widely discussed in the framework of XML access control standardization activities. The aim of this paper is to describe a model combining the advantages of these approaches and overcoming their limitations, suitable as the basis of a standard technique for XML access control enforcement. The model specification is given using a Finite State Automata, ensuring generality w.r.t. specific implementation techniques.

(C) 2008 Elsevier B.V. All rights reserved.
\end{abstract}

Keywords: XML; Security enforcement; XPath; XUpdate; DFA

\section{Introduction}

The eXtensible Markup Language (XML) [2] has become the format of choice for data interchange. XML-based systems are now widely deployed in a number of application fields. This success has triggered a growing interest in XML security, and several schemes for XML access control have been proposed. XML access control schemes can be classified in two major categories: node filtering and query rewriting techniques. The first category includes a number of approaches (e.g., $[9,8,19,22]$; for a complete survey, see [19]) that use access policies to compute secure views on XML data sets. User queries are then evaluated on those views. Although views can be prepared off-line, in general, view-based enforcement schemes suffer from high maintenance and storage costs, especially for a large XML repository.

\footnotetext{
* Corresponding author.

E-mail addresses: damiani@dti.unimi.it (E. Damiani), janvier-majirus.fansi@etud.univ-pau.fr (M. Fansi), alban.gabillon@univ-pau.fr (A. Gabillon), marrara@dti.unimi.it (S. Marrara).
}

XML access control via query rewriting ([28,25,27,15,13,4]) has been proposed as a way to remedy these shortcomings. According to this approach, access control rules are not directly applied to the XML dataset to be protected; rather, they are used to translate potentially unsafe user queries into safe ones, to be evaluated against the original XML dataset. Most current proposals translate the policy's access control rules (ACR) to nondeterministic finite automata (NFSA) to rewrite user queries. However, for policies that include many ACRs, NFSA backtrackings may cause unacceptable overhead. Another serious concern is that few of these models provide users with a safe schema representing the information that they are allowed to access. Disclosing the original schema may cause unwanted information leaks. Finally, and perhaps more importantly, NFSA-based models do not specify the details about how implementations should deterministically simulate non-deterministic execution and are therefore not entirely suitable for the specification and standardization of policy enforcement.

On the other hand, a long tradition has been established of using deterministic automata for standardizing protocols and systems implementation-related issues. Besides model checking [26], other important researches and applications 
have described their standards by means of deterministic automata. For instance, ASN.1 (Abstract Syntax Notation One) is a data description language used to define the syntax of communication protocols. It is used widely in both TCP/IP and OSI standard definitions, and is very similar to BNF (Backus-Naur Form).

Another important example of using automata in standard definitions is [34]. This approach deals with the automated generation of a model from an SDL (Specification and Description Language) system specification. In this paper, we describe a Deterministic Finite Automaton (DFA) based query rewriting approach (Section 2) that overcomes the drawbacks of the NFA-based enforcement specifications. The main contributions of this work include:

- A security model based on authorization attributes for XML (Section 2.1) in which the security designer inserts the attributes in the XML schema of the document collection via a GUI. This ensures a policy-dependent view of the XML schema (or annotated schema).

- A formalization based on deterministic automata which ensures a high level of generality (i.e., the automaton can be implemented in different ways) while be detailed enough to be suitable as a basis for standardizing the enforcement technique. From this formalization we straightforwardly derive algorithms for computing the user view of the schema (Section 2.2) and the rewriting DFA (Section 2.3) from the annotated schema.

- A way to exploit the standard operators EXCEPT and UNION of XPath to produce a sound and complete rewriting procedure (Section 2.4) of the user query. Detailed Examples (Section 2.7) illustrate the approach.

- A proof that our approach is sound and complete by means of a formal proof of correctness (Section 2.8). The complexity analysis (Section 2.9) shows that the entire procedure is efficient as it is linear with the size (i.e. the number of element definitions) and the depth of the repository schema.

- An approach to securely handle the commands of the recent XUpdate [24] standard. Namely, the authorization designer annotates the repository schema with some write attributes (insert, update, delete). The annotated schema is afterward translated into a Deterministic Finite Automaton (for updates). Rewriting an XUpdate request into a safe one is done in two steps:

- Whenever a user sends an XUpdate request over her view, we first rewrite the expression selecting the nodes to be updated according to the principles described in Section 3. This step is necessary since the user should not be able to update nodes she is not entitled to see.

- Then, we rewrite the XUpdate command over the DFA for updates in order to obtain a safe query (i.e. a query updating the nodes the user is permitted to update).

Related Work is discussed in Section 4. Finally, Section 5 concludes this paper and discusses future work.

\subsection{XML security and current standards}

A major milestone in the XML security standardization is availability of policy enforcement modules for the eXtensible Access Control Markup Language (XACML). Developed by the OASIS Consortium, XACML is an XML-based markup language to encode access control policies. The policy language is flexible and enables the specification of fine-grained, machine-readable policies that can be used to control access to computational resources, including web services and a variety of digital objects, including XML data streams. A number of XML repositories such as Fedora [17] introduced Authorization modules built upon the Sun XACML engine. Each XACML policy defines:

- a "target" that describes what the policy applies to (by referring to attributes of users, operations, objects, datastreams, dates, and more), and

- one or more "rules" to permit or deny access.

Since the beginning of the XACML ([36]) standardization activity within the OASIS, the XML security community has focused on two important points regarding objects to be protected:

- an access control policy has to define clearly the objects target of the enforcement;

- every object, target of a user's request, has to be compared with the policy in order to outline which rules have to be applied;

When the object to be protected consists of XML data, two different ways of identifying it can be devised:

- use of XPath both to define the target object in the security policy, and to access the object during a request

- use of schema annotations to define the security requirements and of XPath to access the protected data.

The current XPath standard is not the best choice to address $\mathrm{XML}$ objects in a security environment, because it presents some features (e.g. relative path expressions) whose importance and necessity when targeting the objects of access control policies are questionable. ${ }^{1}$ Here we will consider X Path - -

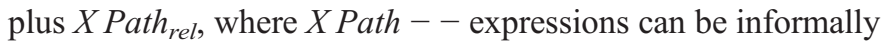
defined as follows:

XPath $--:=\varepsilon|l| *\left|p_{1} / p_{2}\right| / / p_{1} \mid p[q]$ where $p_{1}$ and $p_{2}$ are XPath -- expressions; $\varepsilon, l, *$ denote the empty path, a label and a wildcard, respectively; / and // stand for child-axis and descendant-or-self-axis; and finally, $q$ is called a qualifier. We rewrite the request in the subset $\zeta:=\left\{\varepsilon|l| p_{1} / p_{2} \mid p[q]\right\}$

\footnotetext{
${ }^{1}$ For this reason, our approach hints at breaking the monolithic XPath standard into a multi-layered modular language, where each layer is a welldefined sub-language useful for different purposes. This could be achieved by namespace modularization; however, the details of this decomposition are however outside the scope of this paper.
} 
of XPath - - using the functions union and except. $\zeta$ is XPath - - without descendant-or-self axis (//) and wildcards (*). Obviously we can define $X$ Path $_{r e l}$ as XPath minus $X$ Path - - . A standard definition of X Path - - would provide the XML security community of a common, standard, language to access XML objects in the definition of XML security policies and requests management avoiding the problems given by relative paths. Updating XML data can still be considered a research issue (e.g. see [3,5,24,32,33]); however at least some of the building blocks of a data manipulation language for XML are now firmly in place. XUpdate is an XML-based host language for instructions tailored for update tasks. In other words, it expresses updates as well-formed XML documents; specifically, each update is represented by an xupdate: modifications element. XUpdate has now over a dozen implementations; this relative success is due to the fact that it is easy to understand and simple to implement. XUpdate operations have a required select attribute. The value of this attribute is a XPath expression which selects the nodes to update, referred to as context nodes. Besides updating XML content, XUpdate operations can create and delete entire XML fragments. For instance, the XUpdate command $<$ remove select $=$ '//vehicles' $>$ is to remove from a XML document all fragments whose root is an element named vehicles. XUpdate syntax allows the following types of elements:

- xupdate:insert-before

- xupdate:insert-after

- xupdate:append

- xupdate:update

- xupdate:remove

- xupdate:rename

- xupdate:variable

- xupdate:value-of

- xupdate:if.

The interested reader can refer to [24] for a complete description of the XUpdate Language.

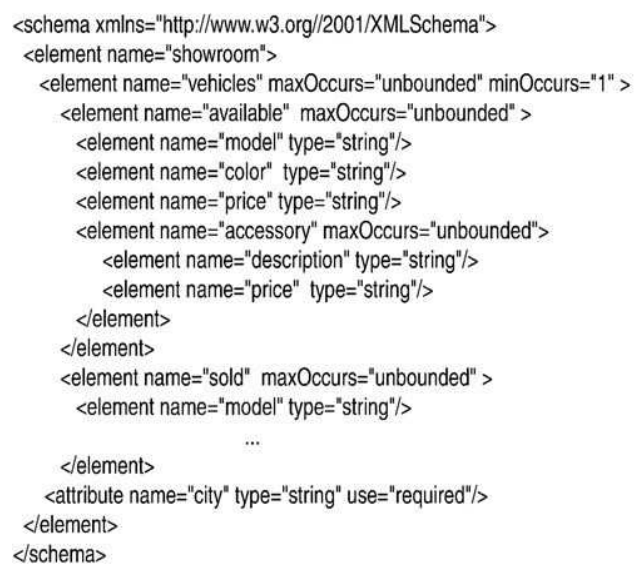

(a)

\section{DFA-based query rewriting}

In this section we present our approach for rewriting potentially unsafe user queries into safe ones. Our technique is based on Deterministic Finite Automata (DFA). We exploit the tree nature of the XML schema to derive the DFA, which is the core of the rewriting procedure. We also outline the proof of correctness for our procedure.

\subsection{Writing the security policy}

The security administrator (SA) uses a Graphical User Interface (GUI) to specify for each user class (role), the part of information that the users are granted or denied access to. Indeed, in order to obtain a policy-dependent view of the schema, the SA annotates the schema using security attributes. This technique was first used in SMOQE [16].

We define the following security attributes: access, condition and dirty. Attribute access specifies the rights of the user on the node. The value of this attribute is either allow or deny. Attribute condition contains a list of predicates that have to evaluate to true for access to be granted. Attribute dirty indicates that some descendants of the current node could be unauthorized. More precisely, a node has a dirty attribute if it has at least one descendant node with either access=deny or a non empty condition attribute attached to it. Annotating the original schema means appending these attributes to element definitions in the schema. The annotated schema is no longer valid w.r.t. the standard W3C XML schema recommendation. It is only an internal representation of the security policy that is never disclosed to the user.

Throughout the rest of this paper, we will consider a repository of XML documents valid w.r.t. the schema depicted in Fig. 1(a) as a working example. In this example, we also consider user Alice and a policy that allows her access to element showroom, conditionally grants her access to elements available and accessory and denies access to sold. Alice is granted access to all other elements (except the descendants of

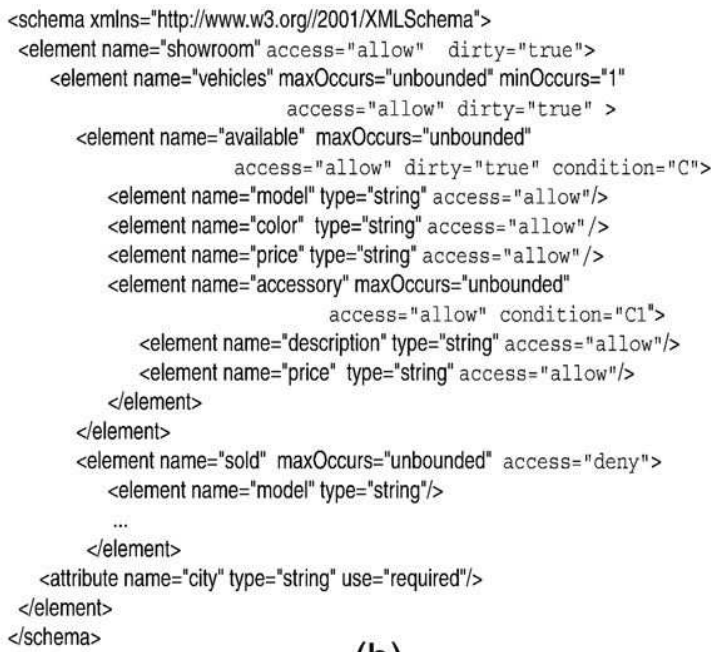

(b)

Fig. 1. The showroom schema (a) and the corresponding annotated schema (b). 
sold of course). The annotated schema is depicted in Fig. 1(b), where security attributes are written in bold.

The remainder of the rewriting procedure, presented in the remaining subsections, consists of three steps:

Step 1 The annotated XML schema is transformed according to the policy that applies to each role. According to her role, the user is provided with the view of the schema (in short $S v$ ) she is entitled to see. Then, she can write her query using information available on $S v$. Henceforth, unless stated otherwise, the term view will refer to the view of the schema and not to the view of a source document.

Step 2 The annotated schema is translated into an automaton which represents the structure of $S v$. Each state within $S v$ contains some security attributes that will further serve us while rewriting the user request.

Step 3 The user query is rewritten using the finite state automaton.

\subsection{Deriving the user view of the schema (Step 1)}

Deriving the user view from the annotated schema is straightforward. We start at the root of the annotated schema tree, and at each element definition, we proceed as follows:

- If the attribute access is allow without any condition then we keep the node as is in the user view.
- If access is allow and there is an attribute condition set then we redefine the node as optional by adding the attribute minOccurs $=0$. In this way if a query fails because the condition is not satisfied, then the user making it will not be able to infer that data have been hidden.

- If access is deny then we discard the sub-tree rooted at the actual node from the user view.

The view for user Alice is depicted in Fig. 2(a).

\subsection{Constructing the automaton (Step 2)}

Constructing the rewriting automaton from the annotated schema is also straightforward. The automaton $M$ derived from the annotated schema consists of an alphabet $\Sigma$, a set of states $S$, a transition function $T: S \times \Sigma \rightarrow S$, a start state $s_{0} \in S$, and a set of accepting states $A \subset S$. The alphabet $\Sigma$ consists of the values of the attributes name of each element definition on the annotated schema.

Creating the states: We start at the root of the annotated schema. The state corresponding to the root (element schema) is $s_{0}$. We create one state for each element definition which has a dirty parent. Indeed, all other nodes (those not dirty) and their subtrees are kept unchanged in the secured view. Hence they do not need to be processed by the automaton. When we encounter a denied node, we create a state for that element and skip the entire sub-tree rooted at that node. Each state $s \in S\left(s \neq s_{0}\right)$ has attributes which represent the security attributes stated at the

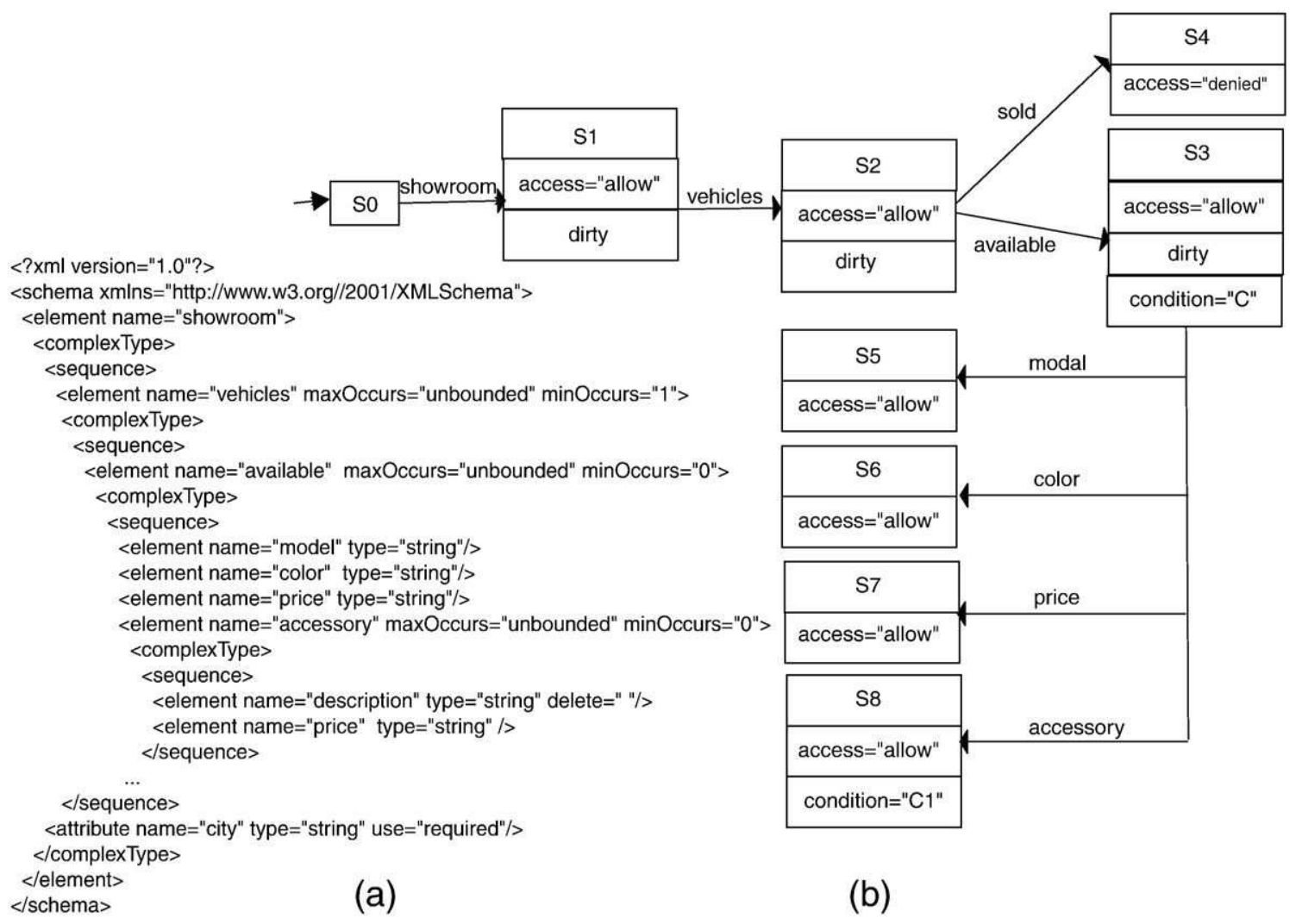

Fig. 2. The user schema view (a) and the rewriting FSA (b). 
corresponding element definition. We give to the state attributes the name and the value of their corresponding security attributes. Each state $s \in S\left(s \neq s_{0}\right)$ is a final state (i.e. $A=S \backslash\left\{s_{0}\right\}$ ).

Defining transitions: There exists a transition from a state $s_{i}$ to a state $s_{j}$ if the element definition corresponding to $s_{i}$ is the parent of the element definition corresponding to $s_{j}$. The transition is labeled by the attribute name of the element definition corresponding to $s_{j}$.

The automaton derived from the annotated schema of Fig. 1(b) is represented in Fig. 2(b).

\subsection{Rewriting the request (Step 3)}

We assume that the user writes her request using XPath $--{ }^{2}$ Hereby, we alleviate the rewriting process overhead since there is no need to backtrack in the automaton. We therefore rewrite the query in two phases. Firstly, we refine the submitted expression and then we rewrite the refined expression via the automaton.

Phase 1: refining the expression. This step consists in refining the request on the basis of the view the user is permitted to see. We first transform the user query (over the repository) to an equivalent one (over the view). Secondly, we execute the latter on user view $(S v)$ and from the target node we go back up to the root node, adding the encountered nodes on the path to form the refined expression. The goal of this procedure is to eliminate every // and $*$ within the expression. As an example, if Alice request is //vehicles/available then the equivalent expression over the view is //element[@name="vehicles"]/ complex Type/sequence/element[@name="avail -able"] and the refined expression is /showroom/vehicles/available. More examples are given in Section 2.7.

Phase 2: Rewriting the request via the automaton. The automaton represents the view the user is permitted to see. Rewriting the user request consists of:

- Processing the first token ${ }^{3}$ of the refined expression

- Moving to the next state of the automaton until either the last token is received, a clean state (i.e., a state that has no attribute dirty) is met or a denied state is encountered.

\subsection{Queries without predicates}

Let us now consider queries without predicates, postponing queries with predicates to the next subsection. After reading the current token, the automaton uses the attributes of the current state and behaves as follows:

- Access is deny. It rejects the request.

- Access is allow.

\footnotetext{
${ }^{2}$ In [20] Gottlob, Koch and Picler show that the loss of expressive power of a fragment like XPath - - w.r.t. XPath is minimal.

${ }^{3}$ We call token a step in the path expression, for example showroom is the first token in /showroom/vehicles/available, while vehicles is the second. / stands for a lookahead.
}

In the latter case, there are two possibilities:

(1) If there is no attribute dirty then the user has the right to consult the entire sub-tree rooted at that node. The token is kept as such, the value of the attribute condition (if any) is attached to the token and the remainder of the source query is appended to the rewritten query. Note that the attribute dirty is for optimizing the rewriting procedure. Indeed, if the access is allow and if there is no attribute dirty then we do not need to analyze the remaining tokens one by one. We can directly append the remainder of the source query to the rewritten query.

(2) If there is the attribute dirty then the token is kept as it is and if there is an attribute condition, its content is attached to the token. Then, the analyzer asks for the next token (if any).

If the last token has been fed into the automaton then we use operator except to eliminate each unauthorized node under the target nodes. If $q$ denotes the rewritten expression after the last token has been fed into the automaton then the final rewritten expression is $q^{\prime}=q$ except $\left(e_{1} \cup e_{2} \cup \ldots \cup e_{n}\right)$, where each $e_{j}$ with $1 \leq j \leq n$ is computed as follows:

The automaton consults one after another the states corresponding to the children of the node represented by the current state. At each state $s$ corresponding to the token $l$, we have the following:

If the attribute access $=$ deny then $l$ is appended to $q$. The result $q / l$ becomes one of the $e_{j}$.

If the attribute access =allow and there is an attribute condition then the negation of the content $C$ of the attribute condition is appended to $l$. The result $l[\operatorname{not}(C)]$ is appended to $q . q / l[\operatorname{not}(C)]$ becomes one of the $e_{j}$. If there is also an attribute dirty then the procedure goes deeper into the automaton (i.e. examines the children of the current token $l$ ) and starts computing another $e_{j}$ with $q$ now being equal to $q / l[C]$. Example $Q_{1}$ in Section 2.7 illustrates this procedure.

\subsection{Queries with predicates}

A query with a predicate is a query which contains a boolean expression. As instance, the expression vehicles[condition] selects the elements vehicles, children of the current node, that satisfy the condition (i.e. the condition evaluation returns TRUE).

In order to simplify the query rewriting process, here we consider only predicates that can be conjunctions or disjunctions of simple expressions $p$ that belong to the set $P$ defined as follows:

$P=\{p \mid p=[\exp ]$ or $p=[\exp$ op val $]\}$, where $\exp \in\left\{\epsilon, l, l_{1} / l_{2}\right\}$, $o p \in\{=, !=,<, \leq,>, \geq\}$ and val is the test value.

Evaluating predicates means to be sure that the user owns the authorization to see the information stored in the nodes that appear in the query predicate $p$.

The query rewriting process is divided into the following steps:

(1) we start saving the predicate expressions appearing in a query $q$ in a correspondence table $=\left\{\left(l_{\mathrm{i}}, p_{\mathrm{i}}\right) \mid p_{\mathrm{i}}\right.$ is a 
predicate and $l_{\mathrm{i}}$ is the node on which the predicate is evaluated, $i=[1 . . n]\}$.

(2) Then we follows the procedure detailed in the previous section to rewrite the query $q$ without the removed predicate expressions. We obtain the rewritten query $q^{\prime}$.

(3) Finally we replace the predicate expressions in the rewritten query $q^{\prime}$ obtaining the query $q^{\prime \prime}$ and construct the automaton as detailed in the previous section. In this case, we stop processing the automaton when a token with predicate(s) is received. We save the current state and check whether the user has the right to consult the nodes that occur within the predicate(s). If she has the right to, we return to the saved state and continue with the next token. Otherwise the request is rejected.

As instance, let us consider the query $q=/ /$ vehicles/available [model="Fiat 500"]/accessory[price $\leq$ "150"]. Following the first step of the procedure, we obtain the correspondence table shown in Fig. 3. After the removal of predicates the query $q$ is //vehicles/available/accessory, which is rewritten into $q^{\prime}=$ /showroom/vehicles/available/accessory.

Then the predicates are inserted again and we obtain $q^{\prime \prime}=$ /showroom/vehicles/available[model= "Fiat 500"]/accessory [price $\leq$ " 150 "]. Then, on the basis of query $q$ " we construct the automaton.

\subsection{Rewriting examples}

Let us now consider the following two queries posed by user Alice. $Q_{1}: / /$ vehicles and $Q_{2}: / /$ vehicles $/ *$ Both queries have to be refined. $Q_{1}$ is transformed to //element [@name="vehicles"] and executed over the view of user Alice. From the target node to the root, we encounter only the definition of element showroom. The request is then refined to $Q_{1}^{\prime}$ : /showroom/vehicles. Likewise $Q_{2}$ is transformed to//element[@name="vehicles"]/complex Type/sequence/ element. The target nodes are the definitions of elements available and sold. Traversing the tree up to the root, we refine $Q_{2}$ as $Q_{2}^{\prime}$ : /showroom/vehicles/available union/showroom/ vehicles/sold. Then, we come to the second phase, i.e., rewriting the refined expression using the automaton. If the refined expression contains the operator union then we rewrite each component of the expression individually and combine the individual results with union to form the global outcome. $Q_{1}^{\prime}$ : /showroom/ vehicles is rewritten as follows: At state $s_{0}$, the automaton receives showroom and reaches state $s_{1}$. State $s_{1}$ indicates via its

\begin{tabular}{|c|c|}
\hline Node & Predicate \\
\hline available & [model="Fiat 500"] \\
\hline accessory & [prices"150"] \\
\hline
\end{tabular}

Fig. 3. Example of correspondence table. attributes that the privilege is allow and some descendants of showroom are inaccessible (attribute dirty). The output at this stage is /showroom. Since $s_{1}$ is dirty, the automaton reads the next token, that is vehicles, leading to state $s_{2}$. This state is allowed but some of its descendants are inaccessible (attribute dirty). We use the operator except to discard all unauthorized nodes. The final result is /showroom/vehicles except (/showroom/vehicles/sold Union/showroom/vehicles/ available[not(C)] Union/showroom/vehicles/available[C]/ accessory $[\operatorname{not}(\mathrm{C} 1)])$ where $\mathrm{C}$ (resp. $\mathrm{C} 1)$ is the condition expressed for the element available (resp. accessory) in the annotated schema (see Fig. 1(b)).

\subsection{Correctness of our query rewriting method}

Proof 1. We show that our query rewriting method is correct by applying the classical the loop invariant [7] technique for proving correctness.

Let us assume that the system receives a user query $x p \in \mathrm{XPath}--$. The first rewriting phase transforms $x p$ to a refined query $q$ which is a set of expression $q_{i} \in \zeta$ joined together with XPath operator union (i.e. $q=q_{1} \cup q_{2} \cup \ldots \cup q_{n}$ ). Phase 2 rewrites in turn each $q_{i}$ into $q_{i}^{\prime}$.

Definition. We say that $q_{i}^{\prime}$ is correct with regard to $q_{i}$, if the result of executing $q_{i}^{\prime}$ over the repository is exactly the same as the answer to $q_{i}$ if $q_{i}$ were executed over the XML repository with access controls correctly enforced.

Let us assume that $q_{i}$ contains $n_{i}$ direct child axis (i.e $q_{i}=/ l_{1} /$ $\left.l_{2} / \ldots / l_{n_{i}}\right)$. Let us call $l_{j} ; j \leq n_{i}$ the current label being processed by the automaton. Let $q_{i(j-1)}^{\prime}=/ l_{1}^{\prime} / l_{2}^{\prime} / \ldots / l_{j-1}^{\prime}$ (note that each $l_{k}^{\prime}$ with $k \leq j-1$ might include a predicate) be the rewritten query of $q_{i_{(j-1)}}=/ l_{1} / l_{2} / \ldots / l_{j-1}$.

We define the following loop invariant: $q_{i_{(j-1)}}^{\prime}$ is correct with regard to $q_{i_{(j-1)}}$.

We show that, this loop invariant holds prior to the first iteration of the second phase of the rewriting procedure, that each iteration maintains the invariant and that the invariant also holds when the procedure terminates.

- Initialization: The loop invariant holds before the first label is fed into the automaton. In fact, prior to the first iteration the rewritten query is $q_{i_{(0)}}^{\prime}=\varepsilon$. This query is obviously correct with regard to $q_{i_{(0)}}=\varepsilon$.

- Maintenance: We show that each iteration maintains the loop invariant. Let us assume that the loop invariant is true before the label $l_{j}$ is received. i.e $q_{i_{(j-1)}}^{\prime}=/ l_{1}^{\prime} / l_{2}^{\prime} / \ldots / l_{j-1}^{\prime}$ is the rewritten path of the sub-expression $/ l_{1} / l_{2} / \ldots / l_{j-1}$ and $q_{i_{(j-1)}}^{\prime}$ is correct with regard to $q_{i_{(j-1)}}$. Let us assume that the current state of the automaton is $s_{j-1}$. According to the first phase of the rewriting procedure, there exists a transition from $s_{j-1}$ on $l_{j}$. Let $s_{j}$ be the state reached by that transition.

Since $q_{i_{(j-1)}}^{\prime}$ is correct regarding $q_{i_{(j-1)}}, q_{i_{(j-1)}}^{\prime}$ returns the same set of nodes $R$ as $q_{i_{(j-1)}}$ would do if it were executed 
over the XML repository with access controls correctly enforced.

When the automaton receives $l_{j}$, it proceeds to the state $s_{j}$ and consults $s_{j}$ 's attributes. If the attribute access is allow then the token is kept as it is. Content $C$ of the attribute condition (if any) is then appended to it.

$q_{i_{(j)}}$ would return all the child nodes $l_{j}$ of the nodes belonging to $R$ for which the user has an authorization. Now, the nodes $l_{j}$ for which the user does not have an authorization are filtered out by the predicate $C$. Therefore, $q_{i_{(j)}}^{\prime}$ returns the same set of nodes as $q_{i_{(j)}}$ would do. Hence, $q_{i_{(j)}}^{\prime}=/ l_{1}^{\prime} / l_{2}^{\prime} / \ldots / l_{j-1}^{\prime} / l j[C]$ is correct regarding $q_{i_{(j)}}=/ l_{1} / l_{2} / \ldots / l_{j}$. If there is no attribute condition, it simply means that the user has an authorization for all the child nodes $l_{j}$ of the nodes belonging to $R$. In that case we also have $q_{i_{(j)}}^{\prime}=/ l_{1}^{\prime} / l_{2}^{\prime} / \ldots / l_{j-1}^{\prime} / l_{j}$ which is correct with regard to $q_{i_{(j)}}=$ $/ l_{1} / l_{2} / \ldots / l_{j}$.

- Termination: The loop terminates in the following cases:

(1) The loop meets a state where access $=$ allow and there is no attribute dirty. Let $s_{j}$ be that state $\left(j \leq n_{i}\right)$. The (possibly empty) remaining path (i.e $/ l_{j+1} / \ldots / l_{n_{i}}$ ) is appended to the already rewritten expression (i.e $\left.2 q_{i_{\left.n_{i}\right)}}^{\prime}=/ l_{1}^{\prime} / l_{2}^{\prime} / \ldots / l_{j-1}^{\prime} / l_{j}^{\prime} / l_{j+1} \ldots / l_{n_{i}}\right)$. As shown in the maintenance step, $q_{i_{(j-1)}}^{\prime}$ is correct with regard to $q_{i_{(j-1)}}$. The absence of the attribute dirty means that the user is allowed to access to the entire sub-trees rooted at nodes $l_{j}^{\prime}$ addressed by $q_{i_{(j)}}^{\prime}$. Thus $q_{i_{\left(n_{i}\right)}}^{\prime}=$ $q_{i_{(j-1)}}^{\prime} / l_{j}^{\prime} / l_{j+1} \ldots / l_{n_{i}}=q_{i}^{\prime}$ returns the same answer as $q_{i_{\left(n_{i}\right)}}=q_{i_{(j-1)}} / l_{j} / l_{j+1} \ldots / l_{n_{i}}=q_{i}$ would do if executed over the XML repository with access controls correctly enforced. Hence $q_{\left(n_{i}\right)}^{\prime}=q_{i}^{\prime}$ is correct with regard to $q_{i_{\left(n_{i}\right)}}=q_{i}$.

(2) The loop meets a state where access $=$ deny. let $s_{j}$ be that state $\left(j \leq n_{i}\right)$. The entire rewritten expression is replaced by the empty $(\varepsilon)$ path.

The fact that access is deny means that the user is forbidden to access any descendant node of the nodes addressed by $q_{i_{(j-1)}^{\prime}}^{\prime}$. Therefore $q_{i_{\left(n_{i}\right)}}=/ l_{1} / l_{2} / \ldots / l_{j} / l_{n_{i}}$ would return the empty set if executed over a repository with access controls correctly enforced. Hence $q^{\prime}=$ $q_{\left(n_{i}\right)}^{\prime}=\varepsilon$ is correct with regard to $q_{i_{\left(n_{i}\right)}}=q_{i}$.

(3) The last token is fed into the automaton:

If access $=$ allow and there is the attribute dirty then it means that some sub-trees of the target nodes cannot be accessed to and, of course, $q_{i_{\left(n_{i}\right)}}$ executed over the XML repository with access controls correctly enforced would not return these sub-trees. Now, for such a case, the rewriting procedure we have defined in Section 2.4 includes a supplementary step which is for filtering out these subtrees by means of the operator except. Therefore we have $q_{\left(n_{i}\right)}^{\prime}=q_{i}^{\prime}$ which is correct with regard to $q_{i_{\left(n_{i}\right)}}=q_{i}$.

Else, termination meets either case (1) or (2).

By applying this proof for each $q_{i}$, we show that $q^{\prime}$ is correct with regard to $q=q_{1} \cup q_{2} \cup \ldots \cup q_{n}$.

\subsection{Complexity analysis}

The complexity of our approach is determined by that of steps 1, 2 and 3 of the rewriting procedure. Let us assume that the repository schema contains $n$ definitions of element nodes. Deriving the user view of the schema (Section 2.2) takes at most $O(n)$ time. Constructing the automaton (Section 2.3) also requires at most $O(n)$ time as well. If $m$ is the depth of the schema, then refining the expression (Section 2.4) takes $O(m)$ time. Since we rewrite the refined expressions by simply traversing the deterministic automaton, this phase takes $O(n)$ time. Hence, the overall time complexity of this proposal is $O(n+m)$.

\section{Updating XML}

Talking about XML security, not only the read privilege needs to be taken into consideration, but also the write privilege plays an important role. In this setting we refer to the XUpdate working draft, and we presented our first results in this direction in [11].

In our model, we consider the following write privileges: DELETE, INSERT, and UPDATE. The semantics of these privileges can be stated as follows:

- if user s holds the INSERT privilege on node $n$ then user $\mathrm{s}$ has the right to add a new sub-tree to node $n$.

- if user s holds the UPDATE privilege on node $n$ then user $\mathrm{s}$ has the right to update node $n$ (i.e., change the values of its immediate children of type text).

- if user s holds the DELETE privilege on node $n$ then user $\mathrm{s}$ has the right to delete the sub-tree of which node $n$ is the root.

Below, for each XUpdate operation we list the write privilege that user s should hold.

Creating node operations. There are three XUpdate instructions for creating XML fragments: insert-before, insert-after and append.

Insert-before inserts a given fragment as the preceding sibling of every context node, and insert-after inserts it as the following sibling of every context node. The operation append allows a node to be created and appended as a child of every context node.

- insert-before/insert-after: user s needs the INSERT privilege on the parent node of every context node.

- Operation append: user s needs the INSERT privilege on every context node.

Update operations. There are two XUpdate instructions for updating XML nodes: update and rename. Operation update can be used to update the content of existing nodes. Operation rename allows an attribute or element node to be renamed after its creation.

- update: if context nodes are elements, then user s needs the UPDATE privilege on the content (text node) of every 
context node. If context nodes are attributes, then user s needs the UPDATE privilege on every context node.

- rename: user s needs the UPDATE privilege on every context node.

Renaming an attribute or updating its value requires the UPDATE privilege on the context node. This choice is consistent with the XPath data model, where an attribute node encapsulates both the attribute and its value. On the contrary, renaming an element requires the UPDATE privilege on the context node and updating its content requires the UPDATE privilege on the content node itself (i.e., the text child of the context node).

Delete operations. There is one XUpdate instruction for deleting XML fragments: remove. Operation remove deletes all sub-trees having a context node as the root. For this operation, user s needs the DELETE privilege on every context node.

\subsection{Securing update operations}

Simply considering the write privileges held by a subject is not sufficient to make XML updates secure. The reason for this can be best understood by considering an analogy with SQL. Let us consider user $A$ A who is the owner of an Employee database table and who has granted to user $_{B} B$ the UPDATE privilege on it. As a result, user_B is not permitted to see user_A's Employee table

SQL $>$ SELECT $*$ FROM user_A.employee;

ERROR ORA-01031: insufficient privilege

but user_B is permitted to update it:

SQL $>$ UPDATE user_A.employee SET salary $=$ salary +100 WHERE salary $>3000 ; 2$ rows updated.

The example above shows that although user_B was not permitted to see user_A's employee table, she was able to learn, through an update command, that there were two employees with a salary greater than 3000 . This is due to the fact that the WHERE clause did perform a read operation on Employee despite the fact that user B did not hold the SELECT privilege on that table. In XUpdate operations the select attribute plays the same role as the WHERE clause in a SQL UPDATE/ DELETE command. Therefore, in order to avoid the inference problem caused by write operations performing read action, we rewrite the XPath expression selecting context nodes according to the principles described in Section 2. Securely controlling an XUpdate operation is then done in two steps.

(1) The XPath expression selecting the context nodes is rewritten according to the read privileges held by the user submitting the XUpdate operation. This step is described in Section 2. It corresponds to the work presented in [12] and uses the DFA for queries. However, when rewriting the XPath expression, we use the answer-as-nodes technique which stipulates that the XPath expression should return the target nodes rather than the entire sub- trees rooted at them. Consequently, we spare the operation except that eliminates forbidden nodes within the sub-tree rooted at the target node.

(2) The XUpdate operation should succeed for the context nodes on which user $s$ holds the proper write privilege and fail for the others. In order to implement this principle, we rewrite a second time the XPath expression selecting the context nodes, so that only the nodes on which user $s$ holds the proper write privilege are selected.

For rewriting the XPath expression according to the write privileges held by the user, we use the following technique: the policy author inserts for each user class (role), the authorization attributes in the XML Schema of the document collection creating the annotated schema. These attributes include insert, update and delete. The value of these attributes is either empty or equal to a list of predicates stating under which conditions the operation should be performed. A sample annotated schema is shown in Fig. 4(b). The annotated schema is afterwards translated into a deterministic finite automaton for updates (see Fig. 4(a)). The automaton traverses its states according to the tokens ${ }^{4}$ of the rewritten expression produced by step 1 , until the last token gets through. Then, the automaton transits to the state corresponding to the target node of the expression. At this position, the finite state machine behaves as follows: ${ }^{5}$

Case 1. The operation is insert-before or insert-after. The automaton backtracks to the previous state, which is the state corresponding to the parent of the context node. Indeed, the user needs the INSERT privilege on the parent node of every context node. If the attribute insert is present at that state then its (possibly empty) value is appended to the XPath expression and returned. If the attribute insert is not present then the expression is rejected.

Case 2. The operation is rename or update. If the attribute update is present then its (possibly empty) value is appended to the XPath expression and returned. If the attribute update is not present then the expression is rejected.

Case 3. The operation is remove. If the attribute delete is present then its (possibly empty) value is appended to the XPath expression and returned. If the attribute delete is not present then the expression is rejected.

Case 4. The operation is append. If the attribute insert is present then its (possibly empty) value is appended to the XPath expression and returned. If the attribute insert is not present then the expression is rejected.

Let us now consider a remove operation on a node $n$. When the user removes node $n$ then she actually deletes the sub-tree rooted on node $n$. Some of the nodes which belong to that subtree may not be visible (i.e. the user may not be permitted to see

\footnotetext{
${ }^{4}$ We call token a step in the path expression, for example showroom is the first token in /showroom/vehicles/available, while vehicles is the second. / stands for a lookahead.

${ }^{5}$ Here, for the sake of simplicity, we consider only commands and privileges addressing element nodes.
} 


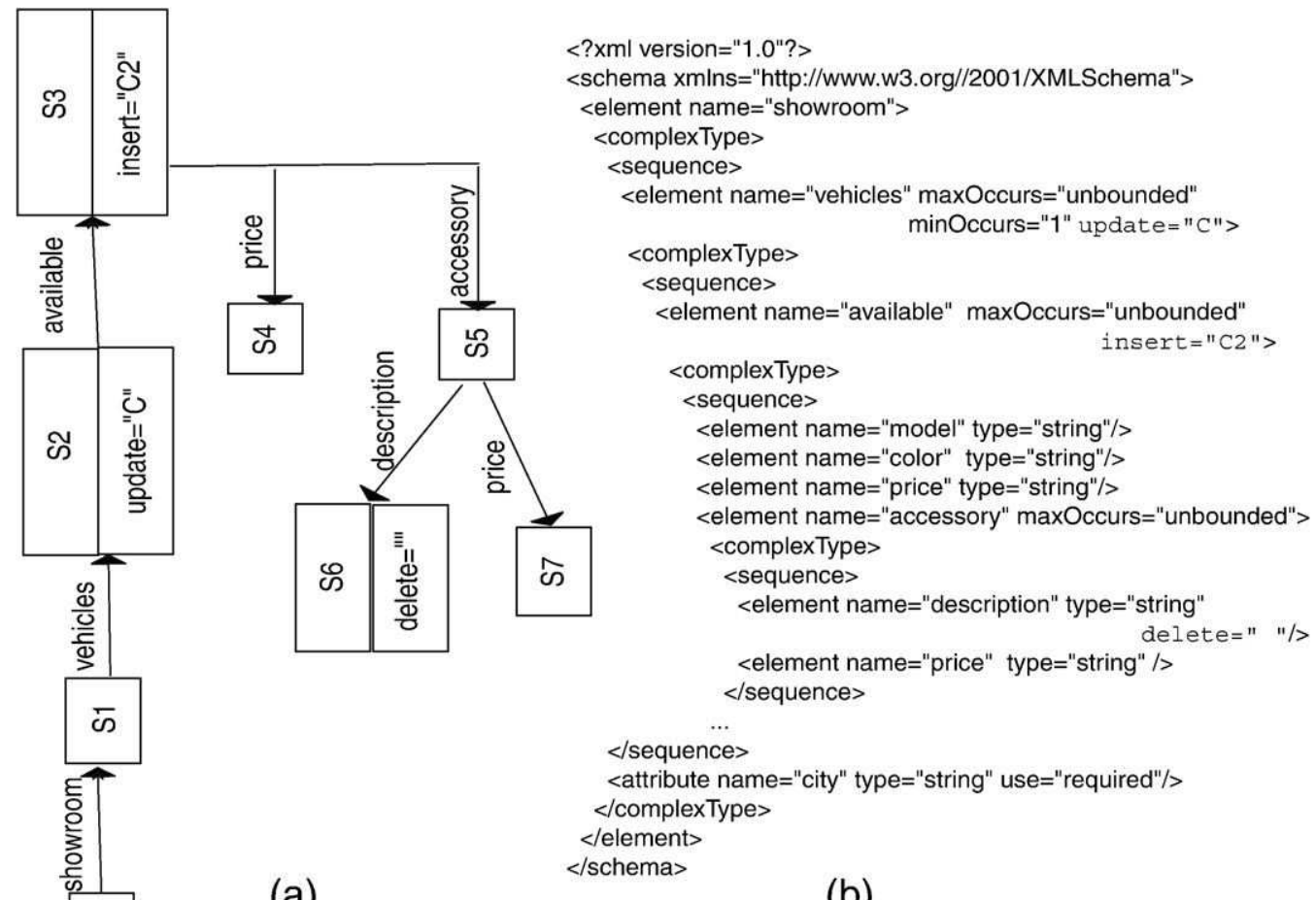

(a)

(b)

Fig. 4. Automaton for updates (a) and write privilege annotated schema (b).

them). Shall we reject the operation if some nodes of the deleted sub-tree do not belong to the user's view? On one hand, this would preserve the integrity of data the user is not permitted to see. On the other hand, it would reveal to the user the existence of data she is not entitled to see. In fact there is no definite answer to this question. This is typically a case of conflict between confidentiality and integrity. Here, we prefer to emphasize the confidentiality, and the command is accepted.

\section{Related work}

In the last few years, several XML access control models have been proposed. After the initial proposal appeared in [9], refinements were described in $[10,22,19,31,8,18]$ ) which use access policies to compute secure views on XML data sets. These models addressed issues like granularity of access, access-control inheritance, overriding, and conflict resolution. All these proposals require provision for view materialization. Although views can be prepared offline, in general, view-based enforcement schemes suffer from high maintenance and storage costs, especially for a large XML repository.

A different approach has been explored in [28], which performs a static analysis that classifies a XML query to be either always-granted or always-denied before submitting it to an XML engine. For partially authorized XML queries, the solution in [28] relies on expensive run-time security checks to filter out the data nodes that users do not have authorizations to access. In [15,23], the problem of unsafe query is solved by rewriting the input query based on the notion of security view. A security view is a restricted view of the document's DTD that exposes the schema structure the user is authorized to use when writing a XPath query. However, in $[15,23]$ there is no control of the query portion under the query target nodes, and forbidden nodes which are descendants of the target ones are disclosed to the requester. Our approach uses the XPath operator EXCEPT to filter out those conflicting portions from the input query.

QFilter [25] is an NFA-based query rewriting technique for XML. Authors in [25] constructs one NFA for each ACR and for each role. Thus this approach can be very inefficient for rewriting queries with "//" axis because of the many backtrackings in the Automaton. This claim is confirmed by the complexity analysis done in [25] which shows that queries with "//" and " $* "$ dramatically aggravates the access control overhead. Also when the input query has predicates in it, they are simply appended to the rewritten query and then can cause information leaks. Moreover, [4] shows that QFilter is not correct by deriving from [25] examples of incorrectly rewritten queries. On the contrary, our proposal uses a DFA-based technique which decreases the complexity of the rewriting procedure and always checks whether the user has the right to consult the nodes that occur within the predicates. We proved its correctness in Section 2.8.

Authors in [27] argue that restricting access to relationships is as important as restricting access to nodes. To this aim, [27] introduced a Security Specification Language for XML (SSX). The SSX enforcement algorithm produces a security view schema for each user. XPath queries against security schemata are then rewritten according to the annotations attached to the 
annotated schema. The main drawback of this solution resides in the SSX language itself, which is based on schema manipulation primitives like copy or delete that appear to be unfit to large-scale access control policy specification. Experiments conducted in [27] show that on average, the approach has a performance which is quite similar to that of materialized views.

The approach proposed in [4] includes a two-phase filtering scheme: the first phase selects access control rules that are related to the user query. The second phase modifies an unsafe query into a safe one. This approach is interesting, but relies on underlying relational DBMS. Also the user is provided with the entire DTD and then can infer sensitive information. Our proposal overcomes these shortcomings by carefully computing the user view of the schema. Our DFA-based system is designed for any XML database. We propose the same technique for securely handling XUpdate commands, as described in Section 3.

As far as the DFA-based approach to enforcement specification is concerned, we remark that it has been applied to a number of related fields, including the problem of improving XML processing efficiency. Improvements to XML processing efficiency can be largely divided into three classes: schemaderived parsing, differential parsing, and stack integration. In particular, schema-derived parsing refers to the technique of pre-compiling an available schema in some manner so that documents conforming to the schema are parsed efficiently. The product of the compilation can be either executable code [6] or data structures for a generic parser [14] defined via automata. Of these, the latter is usually preferred in dynamic environments where new schemas may be required to be recognized. The paper [14] presents a new XML parsing method based on deterministic finite state automata (DFA). A DFA generator is described that automatically translates XML schemas to DFAs for efficient parsing of XML documents and SOAP/XML messages. Another important standard that uses automata in its definition is $R A V I$ [30], a set of specifications proposed as a standard for the interchange of audio/visual interactive applications (AVIs). RAVI consists of an interchange format and a set of functional operators (formulation). Protocols for a RAVI system are defined to allow communication between distributed subsystems. Finally, we note that current standards for access control languages that can be used for protecting XML information $([36,29,35])$ lack a standard technique for enforcing policies via secure query rewriting. For instance, XACML allows to write policies in XML stating access authorization to any type of resources, including XML data. However, XACML does not mandate any specific enforcement algorithm, but relies on different specifications of enforcement according to the protected resource type. Our DFA-based approach is general enough to provide a standard semantics for the enforcement of most XACML policies when applied to protect XML information.

\section{Conclusion}

We described a Deterministic Finite Automata (DFA) based approach to rewrite unsafe queries into safe ones, thus avoiding the many backtrackings inherent to NFAs. We highlighted how our approach improves w.r.t. previous works in the area. Also, we prove the correctness of the approach, and show that our technique is linear with the size and the depth of the repository schema. Although our rewriting procedure is theoretically efficient and suggests good performances, experiments remain work to be done. Moreover, our proposal leaves space for further work. Other approaches [21,1] enforce client-based access control to XML. Indeed, in [21] and [1], the document is encrypted at the server side and decrypted at the client side. The input of their system is then XML data and the output is also XML data, while in our approach both the input and output is an XML query. We are investigating the possibility to diminish the workload at the server side by transferring the rewriting procedure at the client side. Finally our DFA-based approach is general enough to specify the enforcement of most XACML policies when applied to protect XML data. We plan to develop this topic in a future paper.

\section{Acknowledgments}

This work was supported in part by the Italian Basic Research Fund (FIRB) within the TEKNE project and by funding from the French ministry for research under "ACI Sécurité Informatique 2003-2006. Projet CASC". Majirus Fansi holds a Ph.D scholarship granted by the "Conseil Général des Landes".

\section{References}

[1] L. Bouganim, F.D. Ngoc, P. Pucheral, Client-based access control management for XML documents, Proc. of the 30th VLDB Conference, 2004.

[2] T. Bray, J. Paoli, C.M. Sperberg-McQueen, eXtensible Markup Language (XML) 1.0, W3C Recommendation 2nd ed., 2000.

[3] E. Bruno, J.L. Matre, E. Murisasco, Extending XQuery with transformation operators, Proc. of the 2003 ACM Symposium on Document Engineering (DocEng 2003), 2003.

[4] C.W. Byun, S. Park, An efficient yet secure XML access control enforcement by safe and correct query modification, Proc. of the 17th International Conference on Database and Expert Systems Applications (DEXA), 2006.

[5] D. Chamberlin, D. Florescu, J. Robie, XQuery update facility, W3C working draft, May 2006.

[6] K. Chiu, W. Lu, A compiler-based approach to schema-specific XML parsing, Proceedings of the First International Workshop on High Performance XML Processing, 2004.

[7] T.H. Cormen, C.E. Leiserson, R.L. Rivest, C. Stein, Introduction to Algorithms, the MIT Press, 2003.

[8] F. Cuppens, N. Cuppens-Boulahia, T. Sans: Protection of relationships in XML documents with the XML-BB model. In Proc. of ICISS2005.

[9] E. Damiani, S. De Capitani di Vimercati, S. Paraboschi, P. Samarati: Securing XML documents. In Proc. of the 2000 International Conference on Extending Database Technology (EDBT2000).

[10] E. Damiani, S. De Capitani di Vimercati, S. Paraboschi, P. Samarati, A fine-grained access control system for XML documents, ACM Trans. Inf. Syst. Secur., vol. 5(2), ACM Press, New York, 2002, pp. 169-202.

[11] E. Damiani, M.J. Fansi, A. Gabillon, S. Marrara, Securely updating XML, Proceedings of KES 2007, Vietri sul Mare (SA), Italy, September 2007.

[12] E. Damiani, M. Fansi, A. Gabillon, S. Marrara, A general approach to securely querying XML, Proc. of the 5th International Workshop on Security in Information Systems (WOSIS 2007), June 2007. 
[13] S. De Capitani di Vimercati, S. Marrara, P. Samarati: An access control for querying XML data. In Proc. of SWS05 workshop.

[14] R. van Engelen: Constructing finite state automata for high performance web services. Submitted to the International conference on Web Services 2007.

[15] W. Fan, C. Chan, M. Garofalakis: Secure XML querying with security views. In Proc. of SIGMOD 2004 Conference, 2004.

[16] W. Fan, F. Geerts, X. Jia, A. Kementsietsidis, SMOQE: a system for providing secure access to XML, Proc. of the 32nd VLDB Conference, 2006.

[17] The Fedora Project. http://fedoraproject.org/.

[18] B. Finance, S. Medjdoub, P. Pucheral, The case for access control on XML relationships, Proc. of CIKM, 2005.

[19] A. Gabillon: A formal access control model for XMl databases. In Proc. of the 2005 VLDB Workshop on Secure Data Management (SDM), 2005.

[20] G. Gottlob, C. Koch, R. Pichler, The complexity of XPath query evaluation, Proc. of the 22nd ACM SIGACT SIGMOD SIGART Symposium on Principles of Database Systems (PODS-02), ACM Press, San Diego, 2003, pp. 179-190.

[21] N. Kodali, D. Wijesekera: Regulating access to SMIL formatted pay-perview movies. In Proc. of the 2002 ACM workshop on XML security, 2002.

[22] M. Kudo, S. Hada, XML document security based on provisional authorization, Proc. of ACM CCS, 2000.

[23] G. Kuper, F. Massaci, N. Rassadko, Generalized XML security views, Proc. of the 10th SACMAT, 2005.

[24] A. Laux, L. Martin, XML update language (XUpdate)xml:db working draft, http://xmldb-org.sourceforge.net/xupdate, 2000.

[25] B. Luo, D. Lee, W. Lee, P. Liu, QFilter: fine-grained run-time XML access control via NFA-based query rewriting, Proc. of CIKM, 2004.
[26] P. Mazzoni: Model checking tutorial. Technical Report, Politecnico di Milano, Italy. http://www.elet.polimi.it/upload/ghezzi/_PRIVATE/DodCheckMazzoni. pdf.

[27] S. Mohan, A. Sengupta, Y. Wu, J. Klinginsmith, Access Control for XML a dynamic query rewriting approach. In Proc. of VLDB 2005 Conference, 2005.

[28] M. Murata, A. Tozawa, M. Kudo, XML access control using static analysis, Proc. of CCS, 2003.

[29] http://www.nist.gov/.

[30] F. Oguet, C. Schwartz, F. Kretz, M. Quere, RAVI, a proposed standard for the interchange of audio/visualinteractive applications, Selected Areas in Communications, IEEE Journal, vol. 8(Issue 3), Apr 1990, pp. 428-436.

[31] A. Stoica, C. Farkas, Secure XML views, Proc. of the 16th IFIP WG11.3 Working Conference on Database and Application Security, 2002.

[32] G.M. Sur, J. Hammer, J. Simeon, Updatex-an XQuery-based language for processing updates in XML, Proc. of the 2004 International Workshop on Programming Language Technologies for XML (PLAN-XML), 2004.

[33] I. Tatarinov, Z.G. Yves, A.Y. Halevy, D.S. Weld, Updating XML, Proc. of ACM SIGMOD, 2001.

[34] B. Vlaovic, A. Vreea, Z. Brezoc, T. Kapusa, Automated generation of Promela model from SDL specification, Comput. Stand. Interfaces 29 (4) (May 2007) 449-461.

[35] The Web Services Policy Framework. http://www.ibm.com/developerworks/ library/specification/ws-polfram/.

[36] XACML web site. http://xml.coverpages.org/xacml.html. 